Dan R. Thompson

\title{
"No escalation of treatment" as a routine strategy for decision-making in the ICU: pro
}

Received: 21 July 2014

Accepted: 23 July 2014

Published online: 5 August 2014

(C) Springer-Verlag Berlin Heidelberg and ESICM 2014

For a contrasting viewpoint, please go to

doi:10.1007/s00134-014-3421-6.

D. R. Thompson (匹)

Alden March Bioethics Institute, Albany Medical College, 47 New Scotland Avenue, MC162, Albany, NY 12208, USA

e-mail: thompsdr@mail.amc.edu

Tel.: 518-262-5099

The recent article by Morgan et al. entitled "Defining the practice of "no escalation of care' in ICU" has engendered some controversy along with the editorial that accompanied it $[1,2]$. The correct term should be "no escalation of therapy" (NEOT) as stated in the editorial because we always care about the patient. Almost simultaneously Curtis et al. [3] reported an article entitled "The importance of word choice in the care of critically ill patients and their families." The term "no escalation of treatment" is espoused to be particularly confusing to patients and family and from clinician to clinician and should not be used. The term is not more confusing than many of the other terms used in limitations of therapy. Two of the best examples of confusing terminology are DNR (do not resuscitate) and comfort measures. What is it that we do not resuscitate? Usually we do not resuscitate the patient if they have a cardiac arrest. But do we intubate the patient if they have respiratory failure or do we try to prevent the cardiac arrest with intubation? Do we give antiarrhythmic drugs to prevent cardiac arrest? What if the patient has an MI and simple defibrillation without CPR will reverse the problem and leave no longterm impairment? Curtis also suggests that we should not "break up" the advanced cardiovascular life support (ACLS) into components (chest compressions, drugs, defibrillation, and intubation) [4]. It does not make any sense to be doing chest compression in an unintubated patient or giving drugs but no chest compressions to circulate the drugs. However, these terms mean difference things to different people. Some have suggested do not attempt resuscitation (DNAR) or all but cardiac resuscitation and do not intubate (DNI) as some examples of alternate phrases and abbreviations. Perhaps in some cases we should not use the terms because of the lack of clarity, but we do use these terms.

Is it different from NEOT? Morgan gives a pretty good definition for NEOT that is as intuitive as the definition of DNR: "withholding new therapies while continuing current ones." Admittedly, this still leaves some room for interpretation. MOLST and POLST forms have a series of questions addressing what will not be done and both paper and electronic records could have a checklist to be filled out adding to the clarification of what is entailed. Notes about the meeting during which options and limitations are discussed may also document more specific treatments to be included or excluded in the patient's plan of care. Notes about the meetings were things that Morgan admitted were missing frequently from the chart during their review. Making the parameters more apparent will help the whole team and families understand what is intended by the term.

So why have another level of care in the end-of-life decision-making process? Because one size does not fit all, as Curtis comments in the article in Virtual Mentor [5]. At times the step between DNR and comfort measures is too wide for some to come to agreement with. Generally, the concept of shared decision-making is the best way to come to an agreement [6]. At times shared decision-making is difficult to achieve, as we know from practice, and from the literature we also see that not all patients, surrogates, or physicians can agree on the 
concept of shared decision-making all the time [7-9]. We cannot get to yes. There are differences in how patients, surrogates, and physicians may feel about withholding or withdrawing therapy. While we usually think about this as a personalized issue there is a clear difference on a religious basis on withdrawing or withholding $[10,11]$. Sprung's study showed that there was a clear difference between physicians in withholding and withdrawing based on religion and geography and the same is true of patients and surrogates. DNR is about withholding but in comfort measures we usually have at least a component of withdrawal that may not be religiously or culturally acceptable to either party $[12,13]$. This was in particular significant for Jewish, Greek orthodox, or Muslim physicians in Sprung's paper and is similar with patients and surrogates. From a religious standpoint patients and families may have difficulties with withdrawing therapy but may be able to accept not adding further therapy. Some other ethnic groups may also have difficulty with withdrawal [14].

So how do we reach an agreement, or get to a yes, in which caregivers and patient and family can concur if both caregivers and patients and families have problems with some aspect of the continuum? The book Getting to Yes gives us some guidelines to think about bridging this gap [15]. Fisher and his colleagues suggest several steps that may be useful in this situation: listen to yourself and the other stakeholders; for the patient and family, step to their side and see what they see; focus on interests of what is behind your position and their position; invent options and think out of the box-NEOT is one of those out of the box options; use objective criteria (document), sometimes external pressures will not allow more conventional approaches (religious and personal feelings); develop your "best alternative to a negotiated agreement" (BATNA) which respects the patient and their beliefs and build a golden bridge that meets needs and brings the two groups together. Having only two choices does not always work because of personal opinion, religion, and ethnicity. A compromise between the two ends of the spectrum of therapy may make sense and actually be acceptable to all parties. It clearly is not the only answer but the possibility can be useful in doing what is right for the patient. The definition of limited therapy obviously needs to be clearly documented so that everyone-the patient, all the caregivers, and the family-understands. It is important that the caregivers and family recognize that there can be an escalation of comfort measures at the same time as there are limitations to escalation of other therapy. The use of the term "no escalation of therapy" can be useful in the routine discussion of forgoing life support in the ICU.

Conflicts of interest The author declares that he has no conflict of interest.

\section{References}

1. Morgan CK, Varas GM, Pedroza C, Almoosa KF (2014) Defining the practice of "no escalation of care" in the ICU. Crit Care Med 42:357-361

2. Thompson D (2014) Defining an intermediate step in end-of-life therapy. Crit Care Med 42:465-466

3. Curtis JR, Sprung CL, Azoulay E (2014) The importance of word choice in the care of critically ill patients and their families. Intensive Care Med 40:606-608

4. Kross EK, Engelberg RA, Gries CJ, Nielsen EL, Zatzick D, Curtis JR (2011) ICU care associated with symptoms of depression and posttraumatic stress disorder among family members of patients who die in the ICU. Chest 139:795-801

5. Curtis JR (2012) The use of informed assent in withholding cardiopulmonary resuscitation in the ICU. Virtual Mentor $14: 545-550$
6. Carlet J, Thijs LG, Antonelli M, Cassell J, Cox P, Hill N, Hinds C, Pimentel JM, Reinhart K, Thompson BT (2004) Challenges in end-of-life care in the ICU: statement of the 5th international consensus conference in critical care: Brussels, Belgium. Intensive Care Med 30:770-784

7. Curtis JR, Tonelli MR (2011) Shared decision-making in the ICU. Am J Respir Crit Care Med 183:840-841

8. Kon AA (2010) The shared decisionmaking continuum. JAMA 304:903-904

9. Johnson SK, Bautista CA, Hong SY, Weissfeld L, White DB (2011) An empirical study of surrogates preferred level of control over value-laden life support decisions in intensive care units. Am J Respir Crit Care Med 183:915-921

10. Sprung CL, Maia P, Bulow HH, Ricou B, Armaganidis A, Baras M, Wennberg E, Reinhart K, Cohen SL, Fries DR, Nakos G, Thijs LG (2007) The importance of religious affiliation and culture on end-of-life decisions in European intensive care units. Intensive Care Med 33:1732-1739
11. Wilkinson DJ, Truog RD (2013) The luck of the draw: physician-related variability in end-of-life decisionmaking in intensive care. Intensive Care Med 39:1128-1132

12. Romain M, Sprung CL (2014) End-oflife practices in the intensive care unit: the importance of geography, religion, religious affiliation, and culture. Rambam Maimonides Med J 5:e0003

13. Steinberg A, Sprung CL (2006) The dying patient: new Israeli legislation. Intensive Care Med 32:1234-1237

14. Galanti G (2008) Caring for patients from different cultures. University of Pennsylvania Press, Philadelphia

15. Fisher R, Ury W, Patton B (1991) Getting to yes, negotiating agreement without giving in. Penguin, New York 